\title{
Contemporary Issue
}

\section{Rejected but not dejected: Dealing with an unfavourable decision on a scientific manuscript}

\author{
Maj Gen Madhuri Kanitkar, AVSM, VSM ${ }^{a, b}$ \\ ${ }^{a}$ Dean \& Deputy Commandant, Armed Forces Medical College, Pune 411040, India \\ ${ }^{\mathrm{b}}$ Deputy Chairperson, Medical Journal Armed Forces India, Armed Forces Medical College, Pune 411040, India
}

\section{A R T I C L E I N F O}

Article history:

Received 30 January 2018

Accepted 28 February 2018

Keywords:

Scientific manuscript

Unfavourable decision

Rejection

Publication

\begin{abstract}
A B S T R A C T
Unfavourable decisions and rejections on submitted manuscripts are not uncommon in scholarly publications. Rejection in a particular journal need not be viewed as end of all hopes for aspiring authors. Substantial number of rejected manuscripts find their final place in one or the other journal after suitable revision. As an author, it is extremely important to be familiar with common reasons for unfavourable decision/rejection in order to prevent them or to find solutions.
\end{abstract}

๔ 2018 Published by Elsevier B.V. on behalf of Director General, Armed Forces Medical Services.

\section{Introduction}

Scholarly publications are an important means of showcasing scientific endeavours and are also a surrogate marker for academic credentials particularly for career progression in medical academia. Health professionals in academic institutions, are often required to have a certain minimum mandatory number of original research publications in reputed journals to ensure that their academic credentials are maintained. The necessity for having research publications is thus increasingly crucial in the career of health care professionals. Interestingly, increasing demand for research publications has opened up a plethora of novel means of catering to the need including widely circulated open access journals. Despite an exponential growth in the numbers of biomedical journals in the recent past, it is often difficult to choose an appropriate journal for manuscript submission. The matter becomes further complicated in the presence of an abundance of predatory journals/publishers who are willing to publish indiscriminately at a cost to the prospective author. ${ }^{1}$ Indeed, a naïve author may find it difficult to publish an article in an established journal. This is possibly because of the relatively high rejection rates of such journals ranging from $50 \%$ to $90 \%$. With this background, it is apprehended that prospective authors may get demotivated in their preliminary forays into scientific publishing. However there is a ray of hope behind these seemingly unsavoury rejections. Some of the pros and cons from the author's perspectives, of unfavourable decisions in scholarly journals are discussed in this article.

\section{Rejection before peer review}

A manuscript submitted to a journal goes through several stages of review before finally getting published. Many

E-mail address: madhurikanitkar@rediffmail.com. 
established journals have a process of intense scrutiny by the technical staff before a manuscript is assigned to an editor. It may be very disappointing and discouraging for an author to learn that the manuscript has been returned or even rejected at the pre-peer review stage. Some of the potentially avoidable reasons for adverse decisions on a submitted manuscript include but are not limited to the reasons given in Box $1 .^{2-4}$ Most of these issues can be adequately addressed before submitting a manuscript if the author follows the 'Instructions to authors' available on the journal website. It is advisable for authors to conscientiously peruse the aims and scope as well as detailed instructions to authors of a journal before planning to write a manuscript for any particular journal. Authors must also thoroughly scan a manuscript which is ready for submission and read the same again and again to fine-tune minor issues and errors before submitting the manuscript. Another method would be to let colleagues/fellow authors read the manuscript carefully, assuming the role of a peer reviewer and suggest necessary changes before uploading the manuscript. Particular attention is needed in following the chronology and completeness of documents uploaded. For example, if a journal follows blinded manuscript review system, any document giving details of the authors will result in the manuscript being returned even before going for peer review. Next consideration is for selection of the right journal for the right topic for successful publication. Two options can be considered at this stage. Firstly the author can search for any particular topic in any scientific repository such as PubMed or Scopus and determine which journals have published similar but not the same topic in the recent past. Secondly, if possible the author may find out the acceptance rate in a particular shortlisted journal. Many (not all) journals display their acceptance rates on their website.

Plagiarism is another common cause for rejection of manuscripts before and even during peer review or even retraction after publication. Plagiarism is considered to be one of the most serious forms of scientific misconduct. Most journals have their own method of checking plagiarism in submitted manuscripts. There are many free as well as commercial plagiarism detection tools (PDT) available today. Medical Journal Armed Forces India (MJAFI) has been using a professional plagiarism detection tool called iThenticate since 2014. By and large, PDTs are reasonably effective in detection of textual similarities with the database available to them. It is essential for all authors to be familiar with what constitutes plagiarism as also the various types of plagiarism. It is often wrongly believed that giving a reference entitles an author to use text verbatim

Box 1.

Not within the aims and scope of a particular journal.

Manuscript not in the correct format as per the journal style.

- Incorrectly uploaded and incomplete manuscript. Poorly written manuscript.

- Suspected plagiarism. from a published source. Various aspects of plagiarism, their detection and remedies have been aptly described in one of the earlier issues of this journal. ${ }^{5}$ One of the simplest ways to avoid plagiarism is to write the manuscript without using 'cut copy paste' from available resources. As a reference point for authors to relate to, it must be considered that writing a manuscript is akin to writing an essay type of answer in an examination. When we answer such questions, we use our recall memory and write answers. It is unlikely that any software would detect plagiarism in such answers.

Similarly, during the preparation phase, one may read/ consult extensive resources, but when writing a manuscript it is extremely important not to refer to any resources including an author's own publication so as to ensure that the written manuscript is plagiarism free. It is also important to understand that appropriate in-text citations remain an essential component of ethical manuscript writing.

\section{Issues with reviewers}

Besides the causes mentioned above, there are many reasons why reviewers and finally the editors reject a submitted manuscript. One of the most important and common reason for rejection of a manuscript during or after peer review is relative lack of Novelty/Originality/Uniqueness. If a manuscript lacks this vital aspect, it is unlikely to be accepted in any reputed journal. It is to be appreciated that novelty is not necessarily always path breaking research. A manuscript which repeats already established facts without adding any new information is likely to have a lesser priority for a journal. Another important reason for an unfavourable decision on a manuscript is relative lack of relevance. Any biomedical research publication has to have some relevance in the field to which it belongs. For example, a research paper will have better chance of acceptance in a clinical journal if it has a clear cut bedside (viz. diagnostic or treatment related) implication. Flawed methodology, analysis and conclusion are another reason for rejection of a manuscript. ${ }^{2-4}$ As an author, it is important to ensure that a study is not flawed in any respect. The conclusion should always be supported by the study described without any assumptions. Various causes of outright rejection of a manuscript have been described well in an eleven point questionnaire based survey of 17 editors of psychiatry journals by Parmar et al. ${ }^{6}$

\section{Handling 'need for major revision' decisions}

It is but natural for an author to believe that his/her particular manuscript carries an important message and hence it deserves publication. But many a time, a submitted manuscript does not get priority because of various reasons as described earlier. At this stage, it is important to remember that even the best of manuscripts would have undergone several rounds of revision before acceptance. It would be a rarity rather than a norm that an original article manuscript is accepted without any revision. So, as an author one needs to be mentally prepared for at least 2-3 rounds of revision. 
Whenever an author gets an intimation from the journal that the submitted manuscript needs revision, an author should feel pleased that the manuscript has not been rejected at the first instance itself. Many authors get disturbed by the number of comments and at times the critical language of the reviewers. Authors need to understand that in a double blind peer review system, the reviewer is likely to have offered unbiased comments and there is thus no need to take reviewers' comments personally and emotionally. It is an established fact that reviewers' comments more often than not help the author improve the manuscript significantly. It is hence advisable not to react immediately whenever the information for revision of a manuscript is received. This will help overcome the initial emotional setback if any is perceived. It is important to go through each and every point offered by the reviewer. Quite often it is possible to accede to the reviewers' comments and recommendations one by one. Authors need to maintain their composure and ensure usage of dignified language while replying to the reviewers observations irrespective of the comments. If an author strongly feels that a particular suggestion of a reviewer is incorrect, it can be politely stated with supporting evidence. If some suggestions are not workable in the current scenario, the same may be admitted. By and large, editors and reviewers value honest opinion. While replying to the reviewers' comments, it is advised to do so in a tabular format pointwise even if no changes are made for certain comments. The changes incorporated in the revised manuscript may be highlighted for the ease of the reviewer. Besides time taken in for the review process, author delay is an important cause of long turnaround time of a submitted manuscript.

\section{Rejection - what next?}

As discussed, the first thing to do is to take a pause. There is absolutely no need to feel frustrated and dejected and start doubting one's capability. If an author thinks that the manuscript has a sound scientific basis, there is no need to worry after rejection from a particular journal. After the initial setback is over, the author needs to analyze the reasons for rejection. Most journals would give detailed reviewer comments along with the decision of rejection. If a study is methodologically flawed, possibly nothing much can be done about the manuscript. The raw data may be subjected to further analysis as pointed out by the reviewers/editors to improve the manuscript. If an author feels that necessary changes can be incorporated the same should be done without wasting time to avoid delays in processing.

\section{The silver lining}

The question often asked by an author after an unfavourable decision/rejection is 'Is there any hope for my manuscript?' There have been a number of studies on the possible fate of rejected manuscripts. It should not be a surprise that the majority (ranging from $50 \%$ to $>90 \%$ ) of rejected manuscripts find acceptance in one or the other journal. ${ }^{7-9}$ All that is needed is perseverance.

As a norm, most journals do not re-process a manuscript once rejected by them. The review process of a reputed journal itself can be of benefit to authors to help improve their article even if rejected. The author can improve upon a manuscript and submit it to another journal hoping for a positive outcome.

To conclude, unfavourable decisions/rejections for submitted manuscripts are not uncommon. As an author, it is extremely important to be familiar with common reasons for rejection in order to prevent them or to find solutions.

\section{Conflicts of interest}

The author has none to declare.

\section{R E F E R E N C E S}

1. Das AK. Publish and flourish: take the road less travelled!. Med J Armed Forces India. 2017;73(2):178-180.

2. Ali J. Manuscript rejection: causes and remedies. J Young Pharm. 2010;2(1):3-6.

3. Thrower P. https://www.elsevier.com/connect/ 8-reasons-i-rejected-your-article Accessed 12.01.18.

4. Meyer HS, Durning SJ, Sklar D, Maggio LA. Making the first cut: an analysis of academic medicine editors' reasons for not sending manuscripts out for external peer review. Acad Med. 2017. http://dx.doi.org/10.1097/ACM.0000000000001860.

5. Debnath J. Plagiarism: a silent epidemic in scientific writing reasons, recognition and remedies. Med J Armed Forces India. 2016;72(2):164-167.

6. Parmar A, Sarkar S. Reasons for rejection of manuscripts in psychiatry journals: a survey of editors. Asian J Psychiatr. 2017;28:140-141.

7. Grant WD, Cone DC. If at first you don't succeed: the fate of manuscripts rejected by academic emergency medicine. Acad Emerg Med. 2015;22(10):1213-1217.

8. Cejas C. Analysis of the revision process by American journal of roentgenology reviewers and section editors: metrics of rejected manuscripts and their final disposition. Am J Roentgenol. 2017;208(6):1181-1184.

9. Casnici N, Grimaldo F, Gilbert N, Dondio P, Squazzoni F. Assessing peer review by gauging the fate of rejected manuscripts: the case of the Journal of Artificial Societies and Social Simulation. Scientometrics. 2017;113(1):533-546. 